\title{
Clinical application of carbon nanoparticle lymph node tracer in the VI region lymph node dissection of differentiated thyroid cancer
}

\author{
S.P. Sun, Y. Zhang, Z.Q. Cui, Q. Chen, W. Zhang, C.X. Zhou, P.P. Xie \\ and B.G. Liu \\ Department of Breast \& Thyroid Surgery, \\ Liaocheng People's Hospital of Shandong Province, Liaocheng, China \\ Corresponding author: Y. Zhang \\ E-mail: zhangyang_zhy@yeah.net
}

Genet. Mol. Res. 13 (2): 3432-3437 (2014)

Received February 7, 2013

Accepted July 29, 2013

Published April 30, 2014

DOI http://dx.doi.org/10.4238/2014.April.30.4

\begin{abstract}
The application and clinical significance of carbon nanoparticle lymph tracer in the VI region (central region) lymph node dissection of differentiated thyroid cancer was investigated. Eighty patients with differentiated thyroid cancer were equally divided into the carbon nanoparticle-marked group (ipsilateral thyroid injection) and the control group (no injection). All patients underwent standard primary tumor treatment and VI lymph node dissection. The number of lymph nodes retrieved in the carbon nanoparticle group (mean $=6.725$ pieces, range $=1-13)$ was significantly higher than those retrieved in the control group (mean $=3.6$, range $=1-7 ; \mathrm{P}<0.05)$. The black staining lymph node rate was $69.89 \%$. A significantly higher number of lymph nodes less than $2 \mathrm{~mm}$ were detected in the carbon nanoparticle group $(\mathrm{P}=0.0023)$. The transfer rates and lymph node metastasis rates did not differ significantly between the two groups. The black-staining lymph node metastasis rate was $20.74 \%(39 / 188)$ and the non-staining lymph node metastasis rate was $22.22 \%(18 / 81)$, which were not significantly different $(\mathrm{P}=$
\end{abstract}


0.7856). No parathyroid accidental resection was observed in the carbon nanoparticle group, whereas three cases occurred in the control group $(\mathrm{P}=0.2405)$. In conclusion, carbon nanoparticles show good lymphatic tracer effects, easy identification, increased number of lymph nodes retrieved, more accurate reflection of the VI region lymph node status, and increased accuracy of the clinical stage. These results should help develop reasonable surgery programs and follow-up comprehensive treatments, and can help to reduce the risk of accident parathyroid resection.

Key words: Differentiated thyroid cancer; Carbon nanoparticles; VI region lymph node dissection

\section{INTRODUCTION}

Differentiated thyroid cancer is the most common malignant tumor of the head and neck, and shows a high incidence of regional lymph node metastasis. The metastasis rate of the VI region lymph nodes was reported to be as high as $50-70 \%$ (Yang et al., 2002). Metastases of the VI region lymph nodes have important prognostic value for accurate clinical staging, surgical planning, postoperative treatment, follow-up programs, assessment of recurrence risk, and prognosis (Zhang, 2011). In order to acquire a more accurate reflection of the VI region lymph node status, the carbon nanoparticle lymph node tracer technique was used to mark the VI region lymph nodes to guide the surgical dissection. Randomized patients were selected as the control group to explore the feasibility and value of this approach.

\section{MATERIAL AND METHODS}

\section{Clinical data}

Eighty cases of differentiated thyroid cancer patients who received surgical treatment in the Breast and Thyroid Surgery Department of Liaocheng People's Hospital from June 2011 to August 2012 were enrolled in the study. Twenty-three cases were males and 57 cases were females, with a male to female ratio of 1:2.48. The ages of patients ranged from 25 to 71 years with a median of 43 years. The diagnosis of all patients was confirmed by preoperative fine needle aspiration cytology. Inclusion cases were randomly divided into the carbon nanoparticle and control groups according to the random-number table. Statistical analysis between the two groups of patients was based on the MSKCC risk standard of differentiated thyroid cancer. The two groups of patients showed no significant differences in gender, age, tumor pathological stage, histological grade, or tumor location, allowing accurate comparisons. All cases underwent primary tumor treatment and standardized lymph node dissection of the VI region.

\section{Research methods}

The carbon nanoparticle suspension was provided by Chongqing Lesmills Pharma- 
ceutical Co., Ltd. In this study, the same medical team performed all operations and postoperative pathological anatomical analyses.

\section{Surgical methods}

All patients underwent anesthesia intubation, and were placed supinely with necks hyperextended. A curved incision was made $2 \mathrm{~cm}$ above the neck sternal notch along the striae. The skin, subcutaneous tissue, and platysma were cut through layers. The anterior muscle was separated along the neck white line. The front ipsilateral thyroid was revealed, and the side and rear parts of the thyroid were kept intact to reduce damage to the lymphatic network around the thyroid. Two to four points in the ipsilateral thyroid were selected for injection of the carbon nanoparticle suspension using a skin test needle; the volume of each point injection ranged from 0.1 to $0.2 \mathrm{~mL}$. Withdrawing was performed first to prevent injecting into the blood vessels, the injection speed was slow, and gentle pressure was applied at the needle puncture site to prevent solution leakage. In accordance with our practice (Zhang et al., 2011a,b), the isthmus sectomies and the subtotal contralateral sectomies were performed first. After approximately 15 min, the ipsilateral lobectomy was performed, and then the samples were frozen and sent for further pathological confirmation before dissection of the VI region lymph node.

\section{Lymphadenectomy of the VI region}

The lateral side was cut to the medial border of the carotid artery. After conventional exposure of the recurrent laryngeal nerve on the ipsilateral side, the nodes and adipose tissues were swept around the recurrent laryngeal nerve. Meanwhile, the lymph nodes and adipose tissues around the cricothyroid membrane were swept. The inside stretched to the trachea contralateral edge. Parts of the thymus pole were removed below, and the pretracheal lymph nodes and adipose tissue behind were swept, so that the specimen could be removed completely (Yu, 2006). The resected specimens were frozen and sent for routine pathological examinations.

\section{Lymph node biopsy}

The resected specimens of lymph nodes were carefully checked. The number of lymph nodes with black staining and non-staining were documented in the carbon nanoparticle group and the control group. All metastatic lymph node specimens were confirmed with pathological examinations, and specimens were simultaneously checked for the presence of the parathyroid.

\section{Statistical analysis}

The SPSS13.0 software was used to perform the statistical analysis, and $\mathrm{P}<0.05$ reflected a statistically significant difference.

\section{RESULTS}

All operations went smoothly and all of the resected specimens were sent for pathological examinations. 


\section{Number and size of the detected lymph nodes}

A total of 269 lymph nodes were detected in the carbon nanoparticle group with an average of 6.725 pieces (range $=1-13$ pieces). There were 188 black-staining lymph nodes, representing a staining rate of $69.89 \%$. A total of 144 lymph nodes were detected in the control group with an average of 3.6 pieces (range $=1-7$ pieces). The number of detected lymph nodes in the carbon nanoparticle group was significantly higher than that in the control group $(\mathrm{P}<$ $0.05)$. Ninety-five lymph nodes that were less than $2 \mathrm{~mm}$ in length were detected in the carbon nanoparticle group, accounting for 35.32\% (95/269) of all detected lymph nodes in this group. Thirty lymph nodes less than $2 \mathrm{~mm}$ in length were detected in the control group, accounting for $22.90 \%$ of all lymph nodes (30/144). The difference between the two groups was statistically significant $(\mathrm{P}=0.0023)$.

\section{Lymph node metastasis}

There were 22 and 20 lymph node-positive cases in the carbon nanoparticle group and the control group, respectively, and the metastasis rates were 55.0 and $50.0 \%$, respectively, which was not statistically significant $(\mathrm{P}=0.3711)$. The numbers of detected metastatic lymph nodes in the carbon nanoparticle group and the control group were 57 and 35, respectively, accounting for metastatic lymph node rates of $21.19 \%(57 / 269)$ and $24.31 \%(35 / 144)$; the difference was not statistically significant $(\mathrm{P}=0.4683)$. There were 39 cases of black-staining metastatic lymph nodes, and 18 cases of non-staining metastatic lymph nodes, accounting for metastasis rates of $20.74 \%(39 / 188)$ and $22.22 \%(18 / 81)$, respectively, which did not amount to a statistically significant difference $(\mathrm{P}=0.7856)$.

\section{Accidental parathyroid removal rate}

Pathological examinations revealed no case of accidental parathyroid resection in the 40 surgeries of the carbon nanoparticle group, whereas three cases of accidental parathyroid removal occurred in the control group. With the application of the exact method analysis, the difference was not statistically significant $(\mathrm{P}=0.2405)$.

\section{DISCUSSION}

The VI region lymph nodes are lymphatic fatty tissues located on both sides of the carotid artery inner edge below the hyoid bone and above the sternal notch. The prelaryngeal, paratracheal, and pretracheal lymph nodes are the most common sites of thyroid cancer (Forest et al., 2011). Routine VI lymph node dissection in differentiated thyroid cancer surgery has become the consensus treatment strategy of most experts (Wu, 2010). Some experts believe that the VI region lymph state should be examined first before deciding whether or not the lateral neck lymph nodes should be dissected. VI lymph node-positive patients should undergo the lateral neck lymph node dissection, whereas VI lymph node-negative patients should not. From this perspective, region VI lymph node metastasis has been a decisive factor in the choice of surgical procedures. Although the quantity of region VI lymph nodes is not strictly required, the greater the number detected results in greater accuracy of VI region lymph node status. 
The carbon nanoparticle suspension injection is a nanotechnology that processes ultrafine carbon particles with a uniform size of $21 \mathrm{~nm}$ per particle. It was injected into the foci surrounding the tissue gap, and through penetration, diffusion, and macrophage pinocytosis, rapidly moved into the drainage capillary lymphatic strand and gathered in the corresponding lymph nodes to result in black staining. Therefore, the staining effect of regional draining lymph nodes was transferred in the living body, which achieved the tracer function.

Results of this study showed that the detected number of lymph nodes in the carbon nanoparticle group was significantly higher than that of the control group. There are three potential reasons to explain this difference.

First, there were significantly more lymph nodes less than $2 \mathrm{~mm}$ detected in the carbon nanoparticle group than in the control group, which might indicate that surgeons were able to detect these tiny lymph nodes more readily. Indeed, in the carbon nanoparticle group, two blackstained lymph nodes with $1 \mathrm{~mm}$ in diameter in the deep surface of the recurrent laryngeal nerve were found in one patient, and the pathological test quickly confirmed them as metastatic.

Second, because the lymph node search was based on the traditional manual method of mainly looking at specimens, smaller lymph nodes could easily be missed, which might affect the judgment and assessment of the prognosis. Because the carbon nanoparticles stained the lymph nodes black, they were easy to identify, which can help pathologists find lymph nodes faster.

Finally, the boundary between the VI and VII regions are not always very clear. Therefore, in surgery, the thoracic upper opening and the anterior mediastinum all have anatomical risks, which might cause some surgeons to not complete the surgical dissection. After the application of carbon nanoparticles for lymph node searching, the presence of black-stained lymph nodes in the thoracic upper opening or the anterior mediastinum would allow surgeons to expand the scope of operation in order to clear the black-stained lymph nodes. This would result in an increase in the number of lymph nodes detected.

Although the lymph node metastasis rate and degree did not differ between the carbon nanoparticle group and the control group, 22 metastatic lymph nodes were detected in the carbon nanoparticle group, which was more than detected in the control group. The clearance of metastatic lymph nodes might improve prognosis and reduce the risk of recurrence in certain cases.

The difference in the lymph node metastasis rate between the black-staining and nonblack staining nodes was not statistically significant, suggesting that the black-staining lymph nodes were not associated with metastasis. In addition, this result showed that the carbon nanoparticle lymph node tracer could not be used to identify the presence or absence of metastatic lymph nodes.

In this study, no accidental resection of the parathyroid occurred in the carbon nanoparticle group, whereas there were three cases in the control group. Due to variation in the anatomical location and morphology of the parathyroid, it can be very difficult to distinguish it from the neck fat tissue, lymph nodes, or thyroid nodules. Therefore, intraoperative identification and accurate positioning are challenging. Lymph node tracer technology stains lymph nodes black without staining the parathyroid, which makes it easy to distinguish the two. Although there was no statistically significant difference in accidental resection of the parathyroid between the two groups, this was likely due to the small sample of this study. Nevertheless, the results of the present study suggested that the application of carbon nanoparticle tracer and staining would enable more accurate identification and protection of the parathyroid in VI region lymph node resection. 
In summary, the use of the carbon nanoparticle lymph tracer technique was advantageous and convenient for surgeons performing lymphadenectomies. It increased the number of lymph nodes retrieved, especially smaller lymph nodes that are more difficult to detect in conventional surgery, and thus reflected the lymph node status of the VI region more accurately. As a result, the carbon nanoparticle lymph tracer technique could improve the accuracy of the clinical stage, and help to develop reasonable surgery programs and follow-up comprehensive treatments. Furthermore, it may also reduce the occurrence of accidental parathyroid resection. Therefore, we believe that the carbon nanoparticle tracer technique has value for use in lymphadenectomy of differentiated thyroid cancer in the VI region.

\section{REFERENCES}

Forest VI, Clark JR, Ebrahimi A, Cho EA, et al. (2011). Central compartment dissection in thyroid papillary carcinoma. Ann. Surg. 253: 123-130.

Wu Y (2010). Central lymph node dissection for differentiated thyroid cancer treatment. Chin. J. Practical Surg. 30: 898.

Yang GC, LiVolsi VA and Baloch ZW (2002). Thyroid microcarcinoma: fine-needle aspiration diagnosis and histologic follow-up. Int. J. Surg. Pathol. 10: 133-139.

Yu G (2006). IV region lymph node dissection in papillary thyroid cancer surgery application. Mod. Practical Med. 18: 98-99.

Zhang H (2011). The significance of level VI compartment dissection forthe treatment of papillary thyroid carcinoma. Chin. J. Practical Surg. 31: 391-394.

Zhang Y, Cui ZQ and Sun SP (2011a). The choice and significance of neck lymph node dissection for differentiated thyroid carcinoma. Chin. J. Practical Surg. 31: 414-416.

Zhang Y, Cui ZQ and Sun SP (2011b). Rationale and clinicalapplication of simplified modified radical thyroidectomy for differentiated thyroidcarcinoma. Chin. J .Endocr. Surg. 5: 103-105. 